\title{
Construction of Sports Competition Management System Based on Key Technologies of Internet of Things
}

\author{
Guofang Kuang ${ }^{1, a^{*}}$ and Hongsheng $\mathrm{Xu}^{1}$ \\ ${ }^{1}$ Luoyang Normal University, Luoyang, 471934, China \\ akuangguofang2012@163.com
}

Keywords: Competition Management; Sports; Internet of Things; RFID; Intelligent Sports

\begin{abstract}
This paper first analyzes the key technologies of the Internet of things, including: embedded programmable RF ID technology, sensor and detection technology, cognitive computing and intelligent control technology. This paper also analyzes the sports management system and points out the important function of the sports competition management system. The paper presents construction of sports competition management system based on key technologies of Internet of things. The system is applied to sports competition, combining theory with practice to construct a real-time and reliable management system.
\end{abstract}

\section{Introduction}

Internet of things is the use of radio frequency identification devices, infrared sensors, global positioning system (GPS), laser scanner and other different devices, embedded software and hardware systems and network access system to achieve all kinds of things! Access to the Internet becomes a part of the Internet, thus realizing the interconnection of objects and objects, people and objects, and combining with the Internet to form a huge network system.

Sensor technology is also the key technology in computer applications. As we all know, so far most of the computer processing is digital signals. Since the existence of computers, sensors have been needed to convert analog signals into digital signal computers to process. RFID tags are also a sensor technology. RFID technology is a combination of radio frequency technology and embedded technology [1]. RFID technology in automatic identification, the article logistics management has the broad application prospect.

At the same time, the sports management system cabinet will release to the public information on the opening of fitness venues to the society, fitness coaches (social sports instructors) to find information, online engagement, expert video, fitness knowledge and the most authoritative diversity of information, And through the opening of interactive platforms, such as forums, for fitness lovers to create a professional, practical network platform.

It has one of the industry's most complete professional product lines, ranging from sensors, controllers to cloud computing applications. Products, services, intelligent home, and it is transportation logistics, environmental protection, public safety, intelligent fire, industrial monitoring, personal health and other fields.

The development of the Internet of things is also the result of pervasive computing and ubiquitous network development represented by mobile technology, which not only brings about technological progress, but also promotes the transformation of economic and social formation and innovation form through applied innovation. It shapes the fluid characteristics of the knowledge society, and promotes the formation of the next generation innovation (innovation 2.0) form for the knowledge society. With the development of mobile and wireless technology and Internet of things, innovation pays more attention to user experience, and user experience becomes the core of next generation innovation.

Sports management environment: the sum of all kinds of objective factors that directly or indirectly act and influence and decide the activities of sports management [2]. As an open system, sports management is characterized by seeking coordination and adaptation with the external environment, so as to improve the efficiency of the system and constantly meet the sports demand of social growth. 


\section{Analysis and Research on Key Technologies of Internet of Things}

The Internet of things was mainly used in the management of goods and goods at the beginning of its birth. Its key technologies mainly include RFID technology and network technology. With the expansion of demand, people hope to use the Internet of things to realize the control of things. Based on this, the Internet of things needs a certain degree of intelligence, and can adapt dynamically according to the changes of the surrounding environment. In addition, the development of material technology also extends the functional properties of the Internet of things. In the future, the Internet of things will not only realize the accurate operation of macro objects, but also achieve the accurate control of microscopic objects.

RFID: electronic tags belong to the category of smart cards. The concept of the Internet of things (IOT) is the "enable" technology proposed in 1998 by Professor Ashton, Director of the Auto-ID Center at M.I.T.; sensor networks: detection and integration, including temperature, with the help of a variety of sensors [3]. The network of physical phenomena, such as humidity, pressure, speed, etc., is also one of the main bases for Premier Wen's reference to "perceiving China": the term "M2M" is widely used abroad. Focusing on the interconnection of terminal equipment and centralized control management $\mathrm{X}$-Internet,as is shown by equation(1), where $\mathrm{G}$ is three major communications operators are pushing the M2M concept.

$$
G_{N}=\sum_{k=1}^{8}|R(k+1)-R(k)|=2, R(9)=R(1)
$$

The Internet of things is the highly integrated and comprehensive application of the new generation of information network technology. It is an important direction and driving force of the new industrial revolution, which can foster new economic growth points and promote the transformation and upgrading of industrial structure [4]. It is of great significance to improve the efficiency and level of social management and public service. To develop the Internet of things, we must follow the law of industrial development, correctly handle the relationship between market and government, between overall situation and part, between innovation and cooperation, between development and security.

Another key technology used in the Internet of things is sensing technology. As an important means of obtaining information, it is mainly accomplished by means of sensors, sensor nodes and electronic tags. Integrated miniaturized sensors can be embedded into any object and can be monitored in real time. Then the collected information can be uploaded wirelessly to realize universal sensing. Sensor nodes are capable of sensing, computing and communicating, and can be used for data acquisition, processing and transmission.

The sensor gateway can be used to realize the information convergence, and the communication network technology can be used to make the information can be transmitted over long distance and reach the designated application system smoothly [5]. At present, there are mainly 3GW WLAN / LTEN GPRs in China's wireless communication network, while 4G is still in the pilot stage. M2M platform has certain authentication function, so it can provide necessary terminal management services for customers. At the same time, for different access modes, All of them can be connected to M $2 \mathrm{~m}$ platform smoothly, so they can transmit data more smoothly and conveniently. In addition, the M2M platform also has certain management functions, which mind the effective management of user authentication, data routing and so on. For boss system, it is widely used in the Internet of things business because of its strong billing management function.

The secure and reliable transmission of information in the Internet of things involves two aspects: remote communication and close communication. The telecommunication aspect mainly includes IP Internet 2G / 3G mobile communication, satellite communication, Internet networking, and gateway and so on. Short-range mainly includes WiFi, Bluetooth ZigBeeer RFID and UWB technology.

The current RFID technology can satisfy the user's management of goods, but from the perspective of the development of the Internet of things, the RFID technology, which only has the function of management and simple control, can not meet the complex business processing in the future. In the 
future Internet of things applications, people want things! Be able to sense changes in the environment and make adjustments automatically without manual intervention.

\section{Analysis on the Frame of Competitive Sports Competition Management System}

The national sports management organization manages the individual information and authorization of the sports instructors in the provinces and cities of China, which is also the network way for the provinces and cities to submit the relevant documents. System uses IC card as the identity of each instructor, safe and reliable. According to the registration place of the social sports instructor, the system manages the sports instructor at different levels, and reports and statistics the grading data.

Sports management: the management of certain sports organizations, through the implementation of decision-making, organization, leadership, control, innovation and other functions, to coordinate the activities of sports management objects, to achieve the established objectives of the process of activities. Benefit: the quantity of effect achieved in unit time reflects the utilization of working time and has certain relation with benefit. Benefit is the relation between effective output and its input.

Sports stadiums management system core functional modules: front interface support site reservation, access control module interface brush membership card can verify the membership information, lighting controller can control site lighting, as is shown by equation(2), $\mathrm{P}$ is support visual card, Support the member fingerprint identification and the member short message group sends the function [6].

$$
\begin{aligned}
& \bar{P}^{(\beta)}(m+1 \mid m)=\Phi_{w}(m) P^{(\beta)}(m, M) \Phi_{w}^{T}(m)+\bar{Q}_{w}(m) \\
& =\Phi_{w}(m) P^{(\eta)}(m, M) \Phi_{w}^{T}(m)+\bar{Q}_{w}(m) \\
& =\bar{P}^{(\eta)}(m+1 \mid m)
\end{aligned}
$$

The Gymnasium Operation Management system is to provide the gymnasium operators, managers, customers and service personnel with a high-level intelligent system based on the gymnasium, and provide modern management means for the gymnasium operators. The users of the Gymnasium Operation Management system are mainly the stadiums operation management and service personnel, and the service objects during the competition are mainly the stadiums operation management personnel, the competition management personnel, the service personnel, the usual service object is the training, Fitness and leisure personnel; the design of the Gymnasium Operations Management system pays special attention to the different characteristics and objects of use during and after the game.

Under the social market economic system, sports and international intercourse are becoming more and more frequent, the democratization of sports decision - making and the improvement of the degree of science, this inevitably brings new demands to the development of sports management science . At present, there is an urgent need to strengthen the research on the management system and operation mechanism of sports, so as to improve the overall level of sports management in our country, and establish and perfect the macro - control mechanism of sports management, and establish and perfect the relevant supervision and feedback mechanism. At the same time, the development of sports management science will also have some fundamental changes.

The speed and function of the original OA system cannot meet the existing requirements. Therefore, the Velcro platform of AMT is introduced to manage the online reimbursement and budget control of knowledge management report management expenses through the portal management process.

Build internal integrated collaborative office and knowledge management system. Through the portal management to achieve a single landing between multiple systems; using process management to improve the efficiency of business operations; using knowledge management to establish efficient internal information communication and sharing; through report management, real-time analysis of the operation of the process; Through the expenses online reimbursement and budget control management, real-time monitoring of each department expense reimbursement situation, reasonable control of expenditure, convenient budget control of each center, to achieve the implementation of the department budget clarity. 


\section{Construction of Competitive Sports Competition Management System Based on key Technologies of Internet of Things}

Sensor and detection technology is the core of the Internet of things to realize the perception of external environment and its own state, providing the most original information for the processing, transmission, analysis and feedback of the network system. With the development of technology, traditional sensors are gradually realizing miniaturization, intelligence, information and networking.

The opening of school sports venues to the outside world refers to the use of free time for students, teaching staff and nearby residents on the premise of satisfying the needs of school physical education teaching and student sports activities, ensuring normal teaching order in schools and observing the regulations of campus management. Social sports organizations, organs, enterprises and institutions to open the use of sports fitness activities, as is shown by equation(3) [7]. Make full use of social public resources, solve the lack of facilities and meet the people's growing demand for sports fitness.

$$
c(\alpha)=\frac{\rho^{\prime} \theta^{\prime \prime}-\rho^{\prime \prime} \theta^{\prime}}{\left(\left(\rho^{\prime}\right)^{2}+\left(\theta^{\prime}\right)^{2}\right)^{\frac{3}{2}}}
$$

The embedded programmable RFID technology is based on the existing RFID technology. The algorithm is solidified into the chip. Under the guidance of the high-level strategy, the algorithm automatically adjusts the state of the chip under the guidance of the high-level strategy to achieve the adaptation of the environment. In reference [4], a hardware design method of high-performance networked controller based on embedded technology was proposed, and the experiment of inverted pendulum networked control based on the controller was given. Personalized, adaptable, predictable features of high performance embedded programmable RFID design provides a new idea.

Principles of sports management: based on the understanding of the principles of sports management, caused by the rules of action prescribed by people. The establishment of sports management principle should be based on the objective law, but there are certain human factors, in order to strengthen its binding, generally bring directive and legal nature, is the behavior standard that requires people to follow together. This is the fundamental difference between principles and principles of sports management,as is shown by equation(4).

$$
c_{m-1}(n)=\sum_{j \in z} \tilde{h}(n-2 j) c_{m}(j)+\sum_{j \in z} \tilde{g}(n-2 j) d_{m}(j) \quad, n \in z
$$

Network convergence is originated from the convergence of telecommunication network, telephone network and Internet at the service level. With the increasing of network standards and the increase of network complexity and heterogeneity, it is difficult to provide cross-network services. Network fusion technology aims to bridge the digital gap caused by different standards by merging all kinds of networks at the network level and realize seamless switching between different networks.

The venue reservation module of the gymnasium satisfies the user's different reservation management of different station types in different time periods, and can be booked according to the site or by the time period, in which the period of time is reserved for a short period of time and for a long time. Improve the utilization rate of the site. Access control module: the entrance control module is a unique function management module of gymnasium software. It can connect with the hardware gate and can be used to identify whether the membership card is effective or not. Swipe card can also set up automatic membership card store value and membership card balance, as well as limit the membership card type.

The development of the sports fitness management system is to provide a platform for the government to manage the national community sports clubs. Through the network and national networking, the application, evaluation, personnel status, site facilities, and financial situation of the national community sports fitness clubs, to supervise and manage the development of activities.

The development of the gymnasium operation management system is oriented to the customers, investors, managers, operators, and service providers. It provides a full range of solutions for the 
management of the gymnasium so that the administrators of the gymnasium can effectively master the customer resources, effectively carry out internal management, grasp the operating conditions of the gymnasiums in time and accurately, and provide the operation and management for the gymnasium. At the same time, tools provide operational management mode and concept.

\section{Summary}

The Internet of things is a complex system with uncertain, high hybrid and strong correlation features. In order to adapt to the major application needs such as smart grid, intelligent transportation, intelligent community and so on, build perceptual interaction, Autonomous, efficient and secure Internet of things systems need to study the following basic theories, including physical space and information space of the complex coupling mechanism, the autonomous mechanism of the Internet of things, fusion decision-making, Trusted software modeling and system optimization. The school management system of traditional sports items is to supervise and manage the declaration, evaluation, personnel status, site facilities, fund status and activities of traditional schools at national and provincial levels through the national network.

\section{Acknowledgements}

This paper is supported by Science and Technology Key Project of Henan Province (162102310478), and by School Applied Science and Technology Research Foundation 2017 "Research on the Application of Wushu (routine) Intelligent Competition arrangement system based on SVM-KNN".

\section{References}

[1] Ren Zhiyu, Ren Peiran. Internet of things and EPCR / RFID Technology. Forest Engineering: June 1, 2016:67-69.

[2] Amardeo C, Smrma. G. Identities in the Future In tern et of Things. Wireless PersCommun 2011, 49 ( 5) : 353- 363.

[3] Zhang YF. Internet of things Technology and Application, Communications and Information Technology, 2012.1,50-53

[4] Gustavo G, Mario O, Carlos K. Early in frastructure of an Internet of Things in S paces for Learning. Eighth IEEE International Conference on Advanced Learning Technologies, 2013: 381383.

[5] Kuang Guofang, Construction of Smart Sensor Networks Data System Based on Integration Formalized BCCS Model,Sensors \& Transducers,2013.8.

[6] Palis R, Stephen G, and Will D. Cognitive Control for Robot Task Execution, 2005 IEEE International Workshop on Robots and $\mathrm{H}$ um an Interactive Communication, 2005: 440- 445.

[7] Wang Bao-yun. Summary of Research on Internet of things. Journal of Electronic Measurement and Instruments, 2014,12:1-7. 